\title{
REMNANT SPECKLES IN A HIGHLY CORRECTED CORONAGRAPH
}

\author{
E. E. Bloemhof \\ Jet Propulsion Laboratory, California Institute of Technology, Mail Stop 171-113, 4800 Oak Grove Drive, Pasadena, CA 91109 \\ Received 2004 April 14; accepted 2004 June 8; published 2004 June 18
}

\begin{abstract}
Searches for faint companions to stars may use coronagraphs fed by adaptive optics (AO) systems of very high correction. Sensitivity will be limited by focal plane speckles from residual, uncorrected wave-front errors, so it is important to characterize remnant coronagraphic speckles. A general analysis is presented, illustrated with the classical Lyot coronagraph and the newer four quadrant phase mask scheme. Two kinds of remnant speckles, of distinct symmetry, occur that are closely analogous to those arising in direct imaging at high correction. Properties and typical intensity estimates are presented, which are useful for estimating speckle noise and false companion detection levels and reduction strategies. For realistic parameters describing some current groundbased observations, the novel antisymmetric "pinned" speckles, usually neglected even in direct imaging, are nonnegligible in narrowband short exposures. For parameters appropriate to a space-borne coronagraph on the Hubble Space Telescope, these anomalous speckles are in fact dominant close to the star and thus a potentially significant source of false companion detections.
\end{abstract}

Subject headings: instrumentation: adaptive optics — techniques: high angular resolution techniques: image processing — turbulence

\section{INTRODUCTION}

Ground-based companion searches are hampered by large phase errors due to atmospheric turbulence. In space, incident wave fronts are flat, but imperfect optics and slow deformations of the telescope still produce phase errors that cause a slowly changing halo of focal plane speckles. In either situation, adaptive or active wave-front correction is desirable, and the remnant postcorrection errors set important sensitivity limits ( $\mathrm{Ra}-$ cine et al. 1999). In adaptive imaging at high correction, speckles have distinct symmetries (Rouan et al. 2000; Boccaletti et al. 2002; Sivaramakrishnan et al. 2002, 2003). At sufficiently high correction, exotic speckles "pinned" to or amplified by the diffraction-limited point-spread function (PSF) must dominate (Bloemhof et al. 2001). Occurring in antisymmetric patterns, these contribute no net power over an entire image (Bloemhof 2004) but can produce individual speckles brighter than the more familiar unpinned speckles. Simple intensity estimates have been derived for the two speckle types of greatest physical interest (Bloemhof 2003). For unpinned, symmetric speckles, typical intensities are roughly constant throughout the halo and depend on the Strehl ratio $S$ and the deformable-mirror (DM) actuator density $D / a$, where $D$ is the telescope diameter and $a$ the actuator spacing. The pinned, antisymmetric speckles, which tend to dominate at high $S$ and $D / a$, are brightest on the inner Airy rings.

Companion detection is much enhanced when adaptive optics (AO) systems are coupled to coronagraphs, which substantially attenuate both the core and the rings of the diffractionlimited PSF produced by an on-axis star. In this Letter, Fourier-optical analysis is applied to a highly corrected coronagraph. The behavior of remnant coronagraphic speckles is discussed, and typical intensities estimated. It is found, perhaps surprisingly, that the anomalous pinned linear-term speckles may play a significant role in ground- and space-based observations.

\section{FOURIER-OPTICAL ANALYSIS OF A HIGHLY CORRECTED CORONAGRAPH}

Figure 1 shows a telescope/coronagraph schematic. In the notation of Bloemhof et al. (2001), the telescope is modeled by a real aperture function $A(\xi, \eta)$, where $(\xi, \eta)$ are pupil plane coordinates. Scintillation is neglected. Remnant wave-front errors not corrected by the AO system are represented by the real phase function $\phi(\xi, \eta)$. Optical fields in focal plane FP1 and reimaged pupil $\mathrm{P} 2$ are multiplied by functions $f(x, y)$ and $p(\xi, \eta)$, respectively, representing the effects of masks at those locations; $p$ is generally a round hole in an opaque screen blocking light diverted to the outer rim of the pupil by the focal plane mask, and $f$ may be a spot occulting the central few $\lambda / D$ of the field (as in a Lyot coronagraph) or four quadrants of phase shifts alternately 0 or $\pi$ (as in the four quadrant phase mask, or FQPM). Apodized masks and other variants may be treated by straightforward generalization. Focal and pupil plane fields are related by Fourier transformation (denoted $\mathcal{F}\{\})$; the final image has intensity

$I(x, y)=\left|\mathcal{F}\left\{p(\xi, \eta) \mathcal{F}^{-1}\{f(x, y) \mathcal{F}\{A(\xi, \eta) \exp [i \phi(\xi, \eta)]\}\}\right\}\right|^{2}$.

If the degree of adaptive correction is high, the remnant phase $\phi$ is small: the mean square wave-front phase error across the aperture is $\left\langle\phi^{2}\right\rangle=1-S$, where $S$ is the Strehl ratio. At sufficiently high Strehl, the pupil plane optical field $A \exp (i \phi)$ may be approximated as $A(1+i \phi)$, and the coronagraphic image of the bright on-axis star that produces speckles is

$$
\begin{aligned}
I(x, y) \approx & \left|\mathcal{F}\left\{p \mathcal{F}^{-1}\{f \mathcal{F}\{A(1+i \phi)\}\}\right\}\right|^{2} \\
= & \left|\mathcal{F}\left\{p \mathcal{F}^{-1}\{f \mathcal{F}\{A\}\}\right\}\right|^{2} \\
+ & 2 \operatorname{Re}\left[i\left(\mathcal{F}\left\{p \mathcal{F}^{-1}\{f \mathcal{F}\{A\}\}\right\}\right)^{*}\right. \\
& \left.\times\left(\mathcal{F}\left\{p \mathcal{F}^{-1}\{f \mathcal{F}\{\phi\}\}\right\}\right)\right] \\
+ & \left|\mathcal{F}\left\{p \mathcal{F}^{-1}\{f \mathcal{F}\{\phi\}\}\right\}\right|^{2} .
\end{aligned}
$$




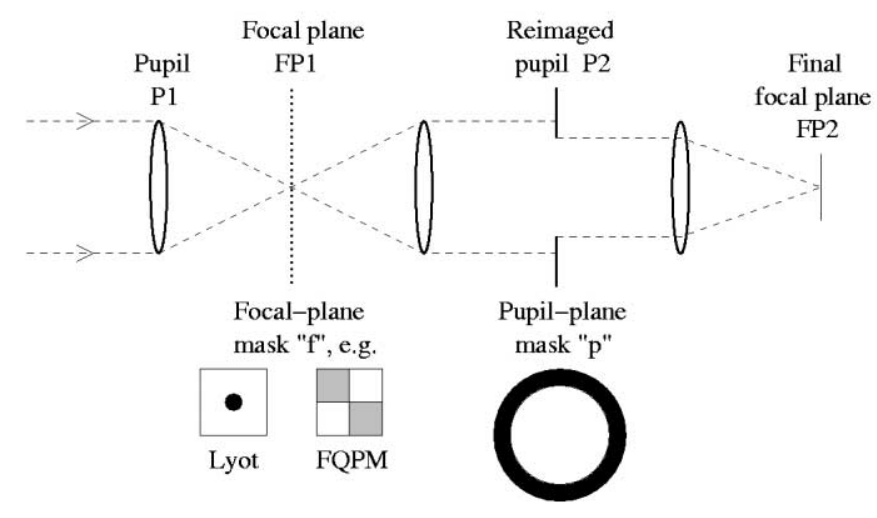

FIG. 1.-Schematic of a coronagraph. Light from a star enters a telescope on the left where the aperture defines the entrance pupil. Image formation is represented schematically by a lens; optical fields in the focal plane are related to those in the pupil plane by Fourier transformation. A focal plane mask "f," inserted as shown, multiplies the fields. A Lyot mask "p" blocks light that the focal plane mask has diverted to the periphery of the reimaged pupil (P2). Final image intensity is the squared modulus of the optical fields.

For simplicity, an unapodized aperture has been assumed, so we may write $A \phi=\phi$. Neglected terms describe fainter speckles that play a lesser role in companion detection $(\S 4)$. An asterisk denotes complex conjugation, and "Re ()" the real part. Equation (2) may be studied numerically, but simple arguments give convenient analytic expressions for the various terms.

The first term is the PSF from the central star (an Airy pattern $|\bar{A}|^{2}, \sim \lambda / D$ wide, if $A$ is an unobscured clear circle of diameter $D$; here the overbar also denotes Fourier transformation) attenuated by the action of the coronagraph. Ideally, attenuation is small for any source a few $\lambda / D$ or more off-axis. Suppression of the main lobe and Airy rings of the on-axis diffraction pattern may be described by

$$
\left|\mathcal{F}\left\{p \mathcal{F}^{-1}\{f \mathcal{F}\{A\}\}\right\}\right|^{2} \sim|\bar{A}|^{2} / R_{\text {cor }},
$$

where the coronagraphic reduction factor $R_{\text {cor }}(x, y)$ (greater than unity and possibly large) is the intensity ratio of the PSF to the coronagraphic image of the on-axis star for each focal plane position $(x, y)$. Azimuthal averages of $R_{\text {cor }}$ may be presented, and averaging over some radial neighborhood may also be appropriate, as the coronagraph will shift Airy rings.

\section{SPATIAL/TEMPORAL PROPERTIES OF CORONAGRAPH SPECKLES}

\subsection{Unpinned Symmetric Speckles from the Quadratic Term}

The third term in equation (2), $\left|\mathcal{F}\left\{p \mathcal{F}^{-1}\{f \mathcal{F}\{\phi\}\}\right\}\right|^{2}$, describes speckles closely analogous to the unpinned quadratic speckles $|\bar{\phi}|^{2}$ derived for simple AO imaging systems, modified only slightly by the coronagraph. Similar nomenclature is appropriate: the term is quadratic in the coronagraph-filtered speckle amplitude, $\mathcal{F}\left\{p \mathcal{F}^{-1}\{f \mathcal{F}\{\phi\}\}\right\}$. These speckles move freely in the focal plane within a halo of diameter $\sim \lambda / a$ and fully account for the speckle power, $1-S$ (Bloemhof 2004). Past studies (Rouan et al. 2000; Boccaletti et al. 2002) considered only the quadratic term, as is appropriate when $R_{\text {cor }}$ is so large that the second term of equation (2) is negligible. (In practice, this is not always the case; see $\S 5$.) These investigators found that quadratic-term speckles appear in spatially symmetric patterns: the phase screen $\phi$ has a Hermitian Fourier transform $\bar{\phi}$ (Bracewell 1986), which is obviously still Hermitian when multiplied by a real Lyot or FQPM focal plane filter function $f$. The inverse transform of this is real, as is the product with the real pupil plane filter function $p$. The final transform $\left(\mathcal{F}\left\{p \mathcal{F}^{-1}\{f \mathcal{F}\{\phi\}\}\right\}\right)$ is then a Hermitian function, whose squared modulus must be symmetric.

Figure 2 demonstrates that quadratic speckles pass through a Lyot or FQPM coronagraph with little alteration: they are slightly broadened and lowered in peak intensity with the narrowing of the reimaged pupil by $p$, as is any off-axis source. These effects, although small $(\sim 10 \%)$, are included in $\S 4$ and seen in numerical simulations. If $f$ describes an apodized mask (e.g., radial Gaussian, radial cosine, linear cosine), it modulates speckle intensities over the focal plane in the obvious way: one may think of individual quadratic speckles as sources from the first focal plane reimaged by the coronagraph.

\subsection{Pinned Antisymmetric Speckles from the Linear Term}

The second term in equation (2), roughly linear in the coronagraphic speckle amplitude $\mathcal{F}\left\{p \mathcal{F}^{-1}\{f \mathcal{F}\{\phi\}\}\right.$, is analogous to the linear term derived for highly corrected imaging systems (Bloemhof et al. 2001) and inherits much of that term's unusual behavior. These speckles are manifestly "pinned" to (localized on) secondary maxima of the primary star's diffraction pattern, inheriting its nulls through the factor $\left(\mathcal{F}\left\{p \mathcal{F}^{-1}\{f \mathcal{F}\{A\}\}\right\}\right)^{*}$. For real aperture function $A, \mathcal{F}\left\{p \mathcal{F}^{-1}\{f \mathcal{F}\{A\}\}\right\}$ is Hermitian by arguments given in $\S 3.1$ for $\mathcal{F}\left\{p \mathcal{F}^{-1}\{f \mathcal{F}\{\phi\}\}\right.$. The product of these two factors is also Hermitian; multiplying by $i$ and extracting the real part give a spatially antisymmetric speckle pattern.

Figure 2 shows that linear-term speckles are attenuated by either a Lyot or FQPM coronagraph. Being spatially antisymmetric, the linear term sums to zero over the entire focal plane for any instantaneous pupil phase realization and so contributes nothing to the speckle power $(1-S)$ (Bloemhof 2004, where zero net power from speckles with possibly large individual intensities was called "anomalous"). Also, in a sufficiently long integration, linear-term speckles will average to zero at any point in the focal plane, assuming the AO system delivers random, zero mean phase errors. On the ground, this averaging ideally takes many cycles of the AO system, each comparable to the atmospheric coherence time. In space, this averaging may take much longer, and there may be more potential for phase excursions.

\section{ESTIMATED INTENSITIES OF CORONAGRAPH SPECKLES}

At high Strehl ratio, simple heuristic arguments may be used to estimate the peak intensity of a typical unpinned speckle from the quadratic term of equation (2):

$$
\begin{aligned}
\text { quadratic } \sim & \left|\mathcal{F}\left\{p \mathcal{F}^{-1}\{f \mathcal{F}\{\phi\}\}\right\}\right|^{2} \sim \frac{(1-S)}{S} \\
& \times(1 / 0.342)(a / D)^{2}\left(D_{p} / D\right)^{2}
\end{aligned}
$$

As in direct imaging (Bloemhof 2003), the speckle image power $1-S$ is divided among $\sim 0.342(D / a)^{2}$ speckles (Roddier 1981). But here the effective pupil diameter is reduced by $D_{p} / D$ (typically $\sim 0.9$ ), broadening the PSF and speckles alike and reducing the peak intensity of a typical speckle by $\left(D_{p} / D\right)^{2}(\sim 0.8)$. As with direct imaging, the factor $S$ in the denominator normalizes the speckle height to that of the corrected PSF, containing fractional power $S$, rather than to that of an ideal PSF; the difference is negligible at high Strehl.

From equation (2), the relative intensity of linear-term speckles 


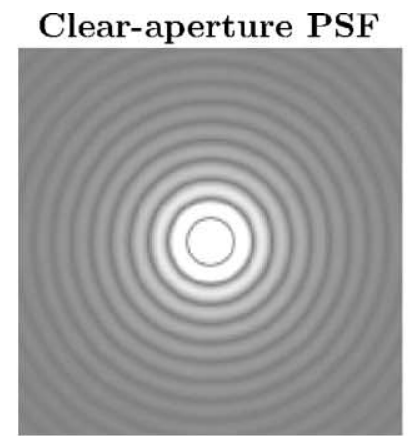

Quadratic, $|\bar{\phi}|^{2}$ : (clear:) Linear, $-2 \operatorname{Im}\{\bar{\phi}\} \bar{A}$ :
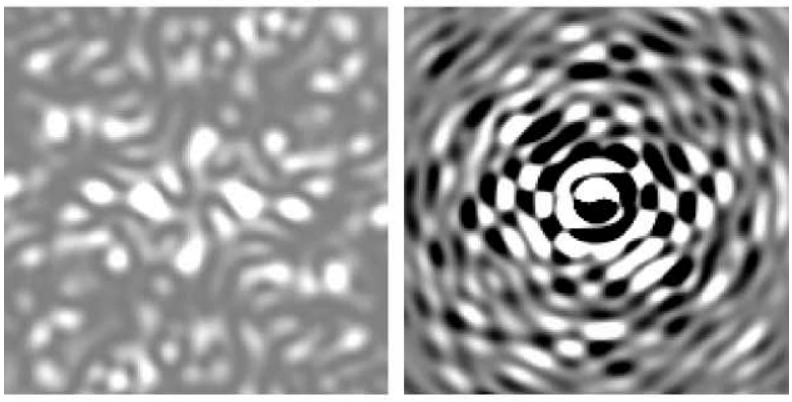

Quadratic:

(thru Lyot:)

Linear:
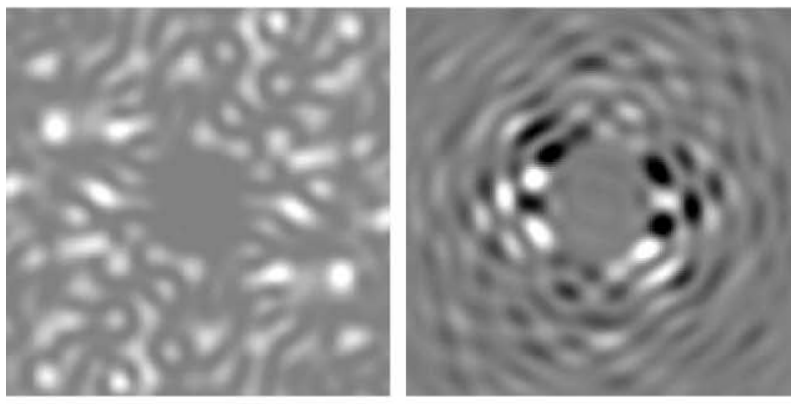

Quadratic:

(thru FQPM:)

Linear:
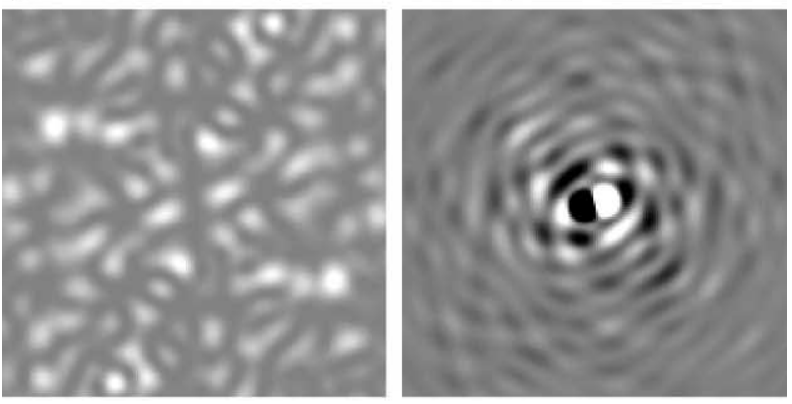

(all for $\mathrm{S}=0.98 ; \mathrm{D} / \mathrm{a}=21$ )

FIG. 2.- Simulated symmetric quadratic-term speckles $\left|\mathcal{F}\left\{p \mathcal{F}^{-1}\{f \mathcal{F}\{\phi\}\}\right\}\right|^{2}$ (left) are almost unchanged by coronagraphs, just slightly broadened and attenuated in peak intensity by the Lyot mask. Antisymmetric linear-term speckles $2 \operatorname{Re}\left[i\left(\mathcal{F}\left\{p \mathcal{F}^{-1}\{f \mathcal{F}\{A\}\}\right\}\right)^{*}\left(\mathcal{F}\left\{p \mathcal{F}^{-1}\{f \mathcal{F}\{\phi\}\}\right\}\right)\right]($ right $)$ are significantly attenuated by a coronagraph. At the top, the telescope PSF shows the spatial scale for all panels. The second row shows quadratic and linear speckles for the telescope alone. The bottom two rows show these speckles after passage through Lyot ( $6 \mathrm{ND}$ diameter occulting disk) and FQPM coronagraphs, each with a Lyot filter $0.90 D$ in diameter. The intensity scale is arbitrary but is the same in all speckle panels. Zero is gray; white and black represent positive and negative intensities. Adaptive correction parameters are representative of $H S T$, but coronagraphic reduction factors $R_{\text {cor }}$ are larger. is determined by the relative strength of the coronagraphically attenuated diffraction amplitude, $\left|\mathcal{F}\left\{p \mathcal{F}^{-1}\{f \mathcal{F}\{A\}\}\right\}\right|$, and the speckle amplitude, $\left|\mathcal{F}\left\{p \mathcal{F}^{-1}\{f \mathcal{F}\{\phi\}\}\right\}\right|$. Using equations (3) and (4), the ratio of typical intensities is

$$
\begin{aligned}
\frac{\text { quadratic }}{\mid \text { linear } \mid} \sim & \frac{\left|\mathcal{F}\left\{p \mathcal{F}^{-1}\{f \mathcal{F}\{\phi\}\}\right\}\right|}{\sqrt{2}\left|\mathcal{F}\left\{p \mathcal{F}^{-1}\{f \mathcal{F}\{A\}\}\right\}\right|} \sim \frac{\sqrt{R_{\text {cor }}}}{|\bar{A}|} \frac{1}{\sqrt{2}} \\
& \times \sqrt{\frac{(1-S)}{S}(1 / 0.342)(a / D)^{2}\left(D_{p} / D\right)^{2}} .
\end{aligned}
$$

The unusual effects of the pinned speckles will appear, at least in narrowband short integrations involving only a few speckle realizations, when the ratio in equation (5) is small compared to unity: i.e., at high Strehl ratio $S$ and actuator density $D / a$. The latter condition is equivalent to relatively smooth phase functions across the pupil or to relatively large halos in the image plane. Linear-term speckles will have lower impact when the coronagraphic reduction factor $R_{\text {cor }}$ is very high, suppressing the Airy rings by which they are amplified.

Formally, additional speckle terms may be derived from higher order expansion of the phase exponential in equation (1). For example, in simple notation appropriate to direct imaging, expansion to second order in $\phi$ gives three new terms: $-\operatorname{Re}\left(\bar{A}^{*} \bar{\phi}^{2}\right), \operatorname{Re}\left(i \bar{\phi}^{*} \bar{\phi}^{2}\right)$, and $\left|\bar{\phi}^{2}\right|^{2} / 4$. These are mathematically interesting but produce speckles fainter than those from the physically most relevant terms, linear and quadratic [2 $\operatorname{Re}\left(i \bar{A}^{*} \bar{\phi}\right)$ and $|\bar{\phi}|^{2}$ in this notation]. The brightest new term, $-\operatorname{Re}\left(\bar{A}^{*} \bar{\phi}^{2}\right)$, generates a PSF correction plus pinned speckles of zero temporal mean that are much fainter at high Strehl than those from the archetypical pinned term $2 \operatorname{Re}\left(i \bar{A}^{*} \bar{\phi}\right)$. Hence, in direct or coronagraphic imaging, and generally with either clear or apodized apertures, the terms retained in equation (2) describe the essential leading-order behavior of the PSF, of pinned speckles, and of unpinned speckles.

\section{IMPLICATIONS}

Speckle evolution generates speckle noise $\sigma=\left(F_{s} / 4\right) \times$ $\left(t / \tau_{0}\right)^{1 / 2}$ (Racine et al. 1999); $\tau_{0}$ is the atmospheric coherence time, for ground-based observations, and $F_{s}$ is the intensity of a typical speckle. So equations (4) and (5) determine the speckle noise contributions of unpinned (quadratic) and pinned (linear) speckles. Some examples follow to illustrate these results.

The Lyot coronagraph on the Palomar AO system has been modeled by Sivaramakrishnan et al. (2001). They find a primary-star reduction factor $R_{\text {cor }} \sim 6$ on the second Airy ring, and $\sim 1$ (no substantial reduction) on the 10th, assuming $S \sim$ 0.79 . Then equation (5), with $D / a \sim 16$, predicts that unpinned speckles are brighter than pinned linear-term speckles by a factor of only 1.3 on the second Airy ring, and of about 4 on the 10th. An FQPM coronagraph on the Very Large Telescope has given $R_{\text {cor }} \sim 2.2$ and $\sim 1$ on the second and 10th Airy rings, for $S=0.3$ (A. Boccaletti et al. 2004, in preparation). Assuming that the correction is increased enough to give speckles of distinct symmetry, and assuming that suppression improved by an order of magnitude, linear-term speckles would still approach the intensity of quadratic to within a factor of 2 , even before any image processing to suppress quadratic speckles. These examples show that, even at the moderate wave-front correction feasible on the ground in the near term, the suppression of practical coronagraphs will not render pinned speckles entirely negligible. 
An illustration of these concepts at very high Strehl, where they are most appropriate, is the Near-Infrared Camera and MultiObject Spectrometer (NICMOS) coronagraph on the Hubble Space Telescope (HST), which provides correction better than $S \sim 0.98$. $H$-band images (Schneider \& Silverstone 2003) reveal an inner halo of diameter $\sim 3^{\prime \prime}$, implying a characteristic transverse scale $a$ of aperture phase errors corresponding to $D / a \sim 21$. These parameters imply remnant quadratic speckle intensities of $1.2 \times 10^{-4}$ of the PSF peak. The coronagraphic reduction factor is $R_{\text {cor }} \sim 10$ just outside the occulting spot, at $\sim 2 \lambda / D$, and drops to $\sim 4$ at $1^{\prime \prime} \sim 7 \lambda / D$. So equation (5) predicts that pinned antisymmetric speckles, from the second term of equation (2), are typically brighter than the symmetric quadratic speckles in any instantaneous realization of the pupil phase screen by a factor of roughly 2.5 just outside the occulting spot, where searches for companions will be concentrated. (They are fainter by a factor of only about 1.2 at $1^{\prime \prime}$.)

Simulations are shown in Figure 3. Subtraction of spatially inverted copies of images (Rouan et al. 2000), to suppress quadratic speckles, would enhance the relative intensity of pinned linear speckles, which survive unchanged. An off-axis point source would leave a half-strength antisymmetric signature, so pinned speckles would mimic companions. Time integration may suppress pinned speckles, which have zero mean over a large number of independent realizations, but speckles in a space-borne observatory may evolve quite slowly.

\section{CONCLUSIONS}

On the ground or in space, remnant focal plane speckles will set limits on image noise and coronagraph contrast. In close analogy to high-Strehl direct imaging, the dominant examples of pinned and unpinned speckle behavior are captured by the last two terms in equation (2), roughly linear and quadratic in the coronagraph-filtered speckle amplitude $\mathcal{F}\left\{p \mathcal{F}^{-1}\{f \mathcal{F}\{\phi\}\}\right\}$. These occur in spatially antisymmetric and symmetric patterns, respectively $(\S 3)$; rough estimates of typical intensities are given in $\S 4$. Quadratic speckles move freely within a halo of diameter $\lambda / a$ and fully account for the traditional speckle image power $1-S$.

Linear-term speckles, not previously considered, are exotic, exhibiting "pinning" to the on-axis diffraction pattern and a vanishing image power over the entire focal plane, although individual speckles can be bright (as in the case of direct imaging; Bloemhof et al. 2001; Bloemhof 2003). They are suppressed by coronagraphy along with the diffraction pattern of

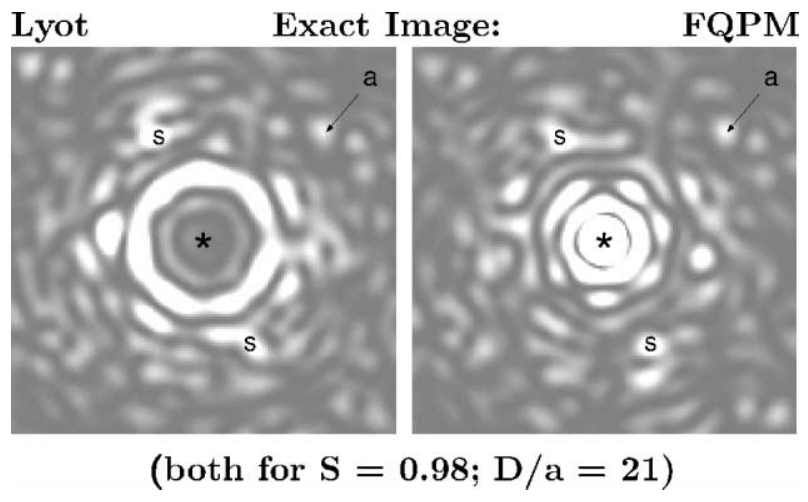

FIG. 3.-Potential false planets in a space-based coronagraph due to linearterm speckles. "Exact" images $\left|\mathcal{F}\left\{p \mathcal{F}^{-1}\{f \mathcal{F}\{A \exp (i \phi)\}\}\right\}\right|^{2}$ are shown for Lyot and FQPM coronagraphs. Many speckles are symmetric (e.g., "s"), but the isolated antisymmetric speckle "a," with no comparably bright counterpart, might produce a false planet detection in a narrowband short integration (see text). Adaptive correction parameters are representative of the HST NICMOS coronagraph, although the model values of $R_{\text {cor }}$ are larger, suppressing antisymmetric linear-term speckles even more than in the real case. Zero is gray; white represents positive intensity.

the bright, on-axis star. However, they become more prominent at very high Strehl ratio $S$ and DM actuator density $D / a$ and therefore can still be appreciable in short, narrowband exposures, particularly in the innermost Airy rings most relevant to companion searches. Linear speckles will average away at any point in the focal plane over many independent speckle realizations, but the timescales involved will be much longer in space than on the ground, and it is less clear that successive phase screens of instrumental origin will have ideal, statistically random behavior. Being antisymmetric, linear speckles will survive the image subtraction that might be used to suppress quadratic-term speckles $(\S 5)$; they would then mimic companions, whose half-power antisymmetric components alone would survive subtraction. So linear speckles may remain a potentially troublesome source of false companion detections that should be considered when space-borne coronagraphic observations are planned.

The research described in this publication was carried out at the Jet Propulsion Laboratory, California Institute of Technology, under a contract with the National Aeronautics and Space Administration.

\section{REFERENCES}

Bloemhof, E. E., Dekany, R. G., Troy, M., \& Oppenheimer, B. R. 2001, ApJ, 558, L71

Bloemhof, E. E. 2003, ApJ, 582, L59

2004, Opt. Lett., 29, 159

Boccaletti, A., Riaud, P., \& Rouan, D. 2002, PASP, 114, 132

Bracewell, R. N. 1986, The Fourier Transform and Its Applications (2nd ed.; New York: McGraw-Hill)

Racine, R., Walker, G. A. H., Nadeau, D., Doyon, R., \& Marois, C. 1999, PASP, 111, 587

Roddier, F. 1981, Prog. Opt., 19, 281
Rouan, D., Riaud, P., Boccaletti, A., Clénet, Y., \& Labeyrie, A. 2000, PASP, 112,1479

Schneider, G., \& Silverstone, M. 2003, Proc. SPIE, 4860, 1

Sivaramakrishnan, A., Hodge, P. E., Makidon, R. B., Perrin, M. D., Lloyd, J. P., Bloemhof, E. E., \& Oppenheimer, B. R. 2003, Proc. SPIE, 4860, 161

Sivaramakrishnan, A., Koresko, C. D., Makidon, R. B., Berkefeld, T., \& Kuchner, M. J. 2001, ApJ, 552, 397

Sivaramakrishnan, A., Lloyd, J. P., Hodge, P. E., \& Macintosh, B. A. 2002, ApJ, 581, L59 